\title{
Necrotizing Enterocolitis, AE
}

National Cancer Institute

\section{Source}

National Cancer Institute. Necrotizing Enterocolitis, AE. NCI Thesaurus. Code C154922.

An adverse event in newborns characterized by extensive mucosal ulceration,

pseudomembrane formation, submucosal hemorrhage, and necrosis in the intestines . 